\title{
Electron self-exchange activation parameters of diethyl sulfide and tetrahydrothiophene
}

\begin{tabular}{|c|c|}
\hline Full Research Paper & Open Access \\
\hline $\begin{array}{l}\text { Address: } \\
{ }^{1} \text { Institut für Chemie, Martin-Luther-Universität Halle-Wittenberg, } \\
\text { Kurt-Mothes-Str. 2, } 06120 \text { Halle/Saale, Germany and }{ }^{2} \text { Merck KGaA, } \\
\text { NMR Spectroscopy, Frankfurter Straße 250, } 64293 \text { Darmstadt, } \\
\text { Germany }\end{array}$ & $\begin{array}{l}\text { Beilstein J. Org. Chem. 2013, 9, 1448-1454. } \\
\text { doi:10.3762/bjoc.9.164 } \\
\text { Received: } 01 \text { May } 2013 \\
\text { Accepted: } 27 \text { June } 2013 \\
\text { Published: } 19 \text { July } 2013\end{array}$ \\
\hline Email: & \\
\hline Martin Goez* - martin.goez@chemie.uni-halle.de & $\begin{array}{l}\text { This article is part of the Thematic Series "New reactive intermediates in } \\
\text { organic chemistry". }\end{array}$ \\
\hline * Corresponding author & Guest Editor: G. Bucher \\
\hline $\begin{array}{l}\text { Keywords: } \\
\text { CIDNP; electron transfer; free radicals; kinetics; photochemistry; } \\
\text { pyrylium salts; self-exchange; sulfides }\end{array}$ & $\begin{array}{l}\text { (C) } 2013 \text { Goez and Vogtherr; licensee Beilstein Institute. } \\
\text { License and terms: see end of document. }\end{array}$ \\
\hline
\end{tabular}

\begin{abstract}
Electron transfer between the title compounds and their radical cations, which were generated by photoinduced electron transfer from the sulfides to excited 2,4,6-triphenylpyrylium cations, was investigated by time-resolved measurements of chemically induced dynamic nuclear polarization (CIDNP) in acetonitrile. The strongly negative activation entropies provide evidence for an associative-dissociative electron exchange involving dimeric radical cations. Despite this mechanistic complication, the free energies of activation were found to be well reproduced by the Marcus theory of electron transfer, with the activation barrier still dominated by solvent reorganization.
\end{abstract}

\section{Introduction}

Single-electron transfer is probably the simplest chemical process of an organic molecule, because usually no full bonds are broken or formed. For this reaction type, the relationship between its thermodynamic driving force and its rate is well understood [1,2]; it only depends on a single parameter for each reagent involved, namely, the activation barrier $\Delta G_{0}^{\neq}$of its selfexchange, e.g.,

$$
\mathbf{D}+\mathbf{D}^{\bullet+} \stackrel{\Delta G_{0}^{\ddagger}}{\rightleftharpoons} \mathbf{D}^{\bullet+}+\mathbf{D}
$$

Observing these key reactions is complicated by the fact that they involve no change of the sample composition. To make the two sides of the reaction equation distinguishable, it is mandatory to label the reagents; then one can measure the transfer rate of the labels to the other species. For fast reactions, nuclear or electron spins are the only labels that can be applied without disturbing the energetics and kinetics. NMR exchange spectroscopy [3] or EPR line broadening experiments [4] employ radiofrequency pulse schemes to generate a suitable magnetization or coherence for this purpose; however, as all such self- 
exchanges by necessity involve radical species, an "automatic" labeling with nonequilibrium magnetizations is also feasible by utilizing the CIDNP [5-10] effect (chemically induced dynamic nuclear polarization).

CIDNP arises through a complex interplay of nuclear-spinselective intersystem crossing and electron-spin-selective reactivity. Its key intermediates are radical pairs $\overline{\mathbf{R}_{1}^{\bullet} \mathbf{R}_{2}^{\bullet}}$, i.e., two radicals possessing a correlation of their electron spins, which originates from the electron spin multiplicity of their precursor. The two radicals of each spin-correlated pair undergo diffusive separations and may reencounter at a later time. During their excursions, the differential precession of their electron spins causes intersystem crossing with a rate that depends on the spin states of their nuclei through the hyperfine interactions; upon reencounter, a chemical reaction serves to distinguish between singlet and triplet pairs (usually, a geminate reaction, i.e., a reaction of the two radicals with each other, is only feasible in the singlet state, whereas the radicals of the triplet pairs escape and ultimately react with other molecules in the sample, i.e., individually). In conjunction, these two processes sort the nuclear spins within the life of the pairs (typically, less than $1 \mathrm{~ns})$ such that polarizations, i.e., nonequilibrium magnetizations, of exactly equal magnitudes but opposite signs are generated in the products from the singlet and triplet exit channels. These experiments are normally carried out in the magnet of an NMR spectrometer, so the polarizations in the diamagnetic products can be directly sampled by a radiofrequency pulse and manifest themselves as anomalous line intensities in the NMR spectrum.

Given an appropriate acceptor/donor combination $\mathbf{A} / \mathbf{D}$, photoinduced electron transfer [11] is an expedient route to radical pairs $\overline{\mathbf{A}^{\bullet-} \mathbf{D}^{\bullet+}}$ by laser flash photolysis. Without further action on the part of the experimenter, the radical pair mechanism then creates opposite polarizations in the geminate products, i.e., in the starting materials $\mathbf{A}$ and $\mathbf{D}$ regenerated by reverse electron transfer, and in the escaping free radicals $\mathbf{A}^{\cdot-}$ and $\mathbf{D}^{\cdot+}$, in other words, labels all four species with their respective polarizations, e.g., $\mathbf{D}_{\uparrow}$ and $\mathbf{D}_{\downarrow}^{\bullet+}$. As was first recognized by Closs [12], the self-exchange leads to a gradual cancelation of the opposite polarizations in each substrate and its corresponding radical ion, e.g.,

$$
\mathbf{D}_{\uparrow}+\mathbf{D}_{\downarrow}^{\bullet+} \rightarrow \mathbf{D}^{\bullet+}+\mathbf{D}
$$

on a timescale of $1 . .100$ microseconds for suitably chosen substrate concentrations. A radiofrequency pulse (typical duration: microseconds for protons) applied at a certain point of time after the laser flash converts the polarizations present in $\mathbf{D}$ at that precise moment into observable coherences and isolates them from the further cancelation, which only operates for magnetizations. Hence, a series of such time-resolved CIDNP experiments [13-16] with different delays between laser flash and observation pulse provides a direct way of measuring the self-exchange rates [17-20]. Practically all published studies of electron self-exchange - apart from indirect determinations based on the Marcus cross relationship [2] - have been carried out on aromatic or olefinic substrates. All known aliphatic radical cations are heteroatom-centered, which considerably increases the mechanistic intricacies. The main complication arises from fast deprotonation of $\mathbf{D}^{\bullet+}$ at the $\alpha$ carbon to give a neutral radical $\mathbf{D}_{-\mathbf{H}}^{\bullet}$, which can occur at the radical-pair stage in a direct reaction (base, $\mathbf{A}^{--}$) or even at any stage of the reaction by a relayed proton transfer (relay base, D) [21-23] and turns the gross reaction $\mathbf{D} \stackrel{-\mathbf{e}^{-}}{\longrightarrow} \mathbf{D}^{\bullet+} \stackrel{-\mathbf{H}^{+}}{\longrightarrow} \mathbf{D}_{-\mathbf{H}}^{\bullet}$ into a hydrogen abstraction. In a previous report on two aliphatic amines [20], we have used time-resolved CIDNP experiments to distinguish between the ensuing electron and hydrogen self-exchanges of $\mathbf{D}^{\bullet+}$ and $\mathbf{D}_{-\mathbf{H}}^{\bullet}$. In the present work, we investigate two structurally similar aliphatic sulfides, diethyl sulfide DES and tetrahydrothiophene THTP, for which we have barred the direct deprotonation at $\mathrm{C}_{\alpha}$ by the choice of a suitable sensitizer; for these substrates, a relayed deprotonation is also impossible because they are not sufficiently basic; however, $\mathbf{D}^{\bullet+}$ can stabilize by dimer formation with surplus $\mathbf{D}$ to give $(\mathbf{D} \therefore \mathbf{D})^{+}[24]$. Except for an order-of-magnitude estimate of the exchange rate from EPR line broadening [25] and a brief remark in an earlier communication by us [26], this is the first report on direct measurements of the electron self-exchange of such sulfurcentered aliphatic radical cations. As we will show, the activation parameters provide evidence that the observed exchange indeed takes place between dimeric radical cations and $\mathbf{D}$.

\section{Results and Discussion}

On the AM1 level, the heats of formation of $\mathbf{D}$ and $\mathbf{D}_{-\mathbf{H}}^{\bullet}$ are $-92 \mathrm{~kJ} / \mathrm{mol}$ and $-14 \mathrm{~kJ} / \mathrm{mol}$ for diethyl sulfide DES as opposed to $-69 \mathrm{~kJ} / \mathrm{mol}$ and $+85 \mathrm{~kJ} / \mathrm{mol}$ for triethylamine, the quencher used in our earlier work [21-23], so for any sensitizer the radical pair containing $\mathbf{D}_{-\mathbf{H}}^{\bullet}$ lies about $75 \mathrm{~kJ} / \mathrm{mol}$ higher above the reactants in the case of the amine. From the oxidation potentials in the literature (DES, 1.65 V [27]; triethylamine, $0.96 \mathrm{~V}$ [21]; both in acetonitrile versus SCE) the opposite order, even with a numerically similar difference, is calculated for the radical pair with $\mathbf{D}^{\bullet+}$, although observations [28,29] that for bifunctional donors comprising both an amine and a sulfide moiety photoinduced electron transfer occurs exclusively from $\mathbf{S}$, not $\mathbf{N}$, cast some doubt on whether these reported potentials are the true equilibrium potentials for these irreversible redox systems. In any case, a sensitizer that is a potent electron 
acceptor but has no hydrogen-abstracting power is mandatory to shift the energetics so as to stop the photoreaction after the stage of the electron transfer.

Two other demands on the sensitizer are imposed by the necessity of maximizing the CIDNP effects, because intrinsically low enhancement factors are expected in consequence [6] of the very high $g$ values of sulfur-centered radical cations. First, to suppress instant electron return and thereby maximize the number of radical pairs available for generating polarizations, a triplet sensitizer should be used [11]; the long life of a triplet excited state is also the only way to meet the conflicting requirements of bringing the self-exchange rates into the observable kinetic range by correspondingly low donor concentrations while still retaining a sufficient amount of quenching. Second, that sensitizer should be positively charged, because this removes the Coulombic attraction between the radicals of the pair, so they can easily separate to a distance where spin mixing becomes effective [30].

All these conditions are fulfilled by the 2,4,6-triphenylpyrylium cation $\mathbf{T T P}^{+}$. Compared to typical carbonyl sensitizers such as benzophenone or xanthone, its reduction potential $(-0.29 \mathrm{~V}$ in acetonitrile versus SCE [31]) is more favorable by more than $2 \mathrm{~V}$, yet its triplet energy (222 kJ/mol [32]) is only lower by less than $90 \mathrm{~kJ} / \mathrm{mol}$. Its first excited singlet state has a lifetime of 2.9 ns [32], so reacts only marginally with the millimolar sulfide concentrations used in our experiments, but its triplet state (intrinsic intersystem-crossing efficiency, 0.48 [32], with evidence for a noticeable increase in the presence of DES by an intermolecular heavy-atom effect [33]) is quenched [34] quantitatively (lifetime, $10 \mu \mathrm{s}$ [32]; diffusion-controlled electron transfer from DES [35]) under our experimental conditions. Protonation of the pyranyl radical TPP' is unknown.

The CIDNP effects observed in the photoreaction of DES sensitized by $\mathbf{T P P}^{+}$in acetonitrile are typical [12] for a selfexchange: Only the starting materials exhibit CIDNP, i.e., no other spin-polarized products are formed; hence, the radicals must ultimately stabilize by reverse electron transfer. In spectra with continuous illumination, the polarizations are only weak whereas time-resolved experiments (Figure 1) reveal that they are much stronger initially but quickly decay to a low residual level; this cancelation is clear evidence for an exchange between the sulfide-derived radical and its parent compound.

Which sulfide-derived intermediate is the source of CIDNP can be concluded from the the polarization pattern, i.e., from the relative polarization intensities of the different protons; ethyl groups are very convenient for this type of analysis [36]. In an

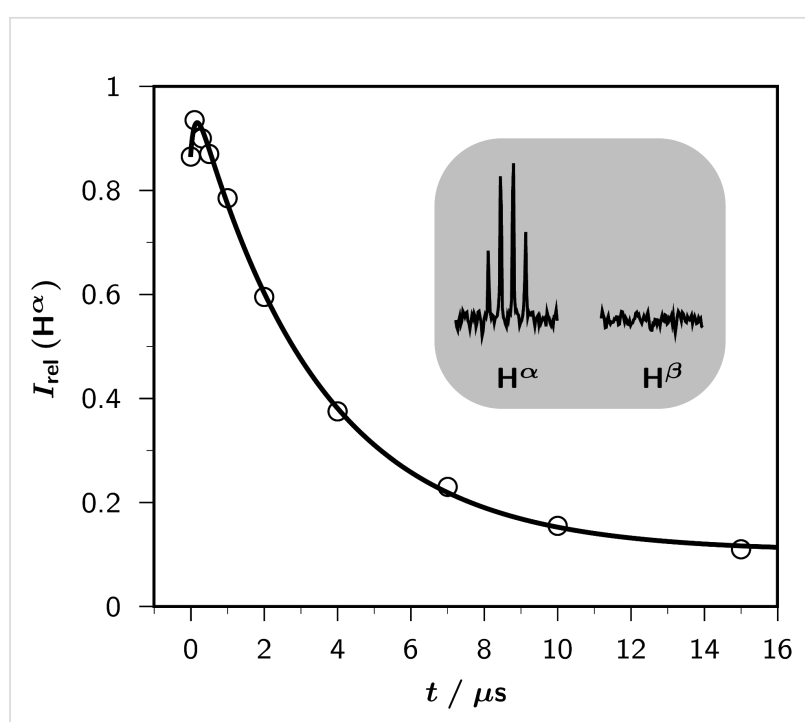

Figure 1: Time-resolved CIDNP measurements on a sample of $5 \times 10^{-3} \mathrm{M}$ DES in acetonitrile- $d_{3}$ sensitized by $2 \times 10^{-4} \mathrm{M} \mathrm{TPP}^{+}$; temperature, $278 \mathrm{~K}$. The main plot shows the relative CIDNP intensities (integrals) $I_{\text {rel }}\left(\mathbf{H}^{\alpha}\right)$ of the $\alpha$ protons of the starting sulfide as functions of the delay $t$ between the laser flash (wavelength, $308 \mathrm{~nm}$ ) and the NMR observation pulse (width, $1 \mu \mathrm{s}$; tip angle, $22.5^{\circ}$ ). The fit function is given by Equation 4, in conjunction with Equation 5 and Equation 6. Best-fit kinetic parameters: $k_{0}, 8.80 \times 10^{6} \mathrm{~s}^{-1}$, corresponding to a quenching rate constant of $1.8 \times 10^{9} \mathrm{M}^{-1} \mathrm{~s}^{-1} ; k_{\mathrm{ex}}, 5.37 \times 10^{7} \mathrm{M}^{-1}$ $\mathrm{s}^{-1} ; T_{1}, 36.5 \mu \mathrm{s}$. The inset displays the actual CIDNP signals of the $\alpha$ and $\beta$ protons of DES $\left(\mathbf{H}^{\alpha}, 2.51 \mathrm{ppm} ; \mathbf{H}^{\beta}, 1.20 \mathrm{ppm}\right)$ at a delay of $0 \mu \mathrm{s}$. Further explanation, see text.

ethyl-substituted heteroatom-centered radical cation, such as DES $^{\bullet+}$, only the $\alpha$ protons experience an appreciable hyperfine coupling, so only these protons can acquire polarizations. In contrast, for an $\alpha$ (heteroatom) substituted ethyl radical, e.g., $\mathbf{D E S}_{-\mathbf{H}}^{\bullet}$, both the $\alpha$ and the $\beta$ protons possess large hyperfine coupling constants, which are negative and positive, respectively. This would translate into a very different polarization pattern, namely, CIDNP signals of similar magnitude but opposite sign for those two types of protons. As the inset of Figure 1 reveals, only the $\alpha$ protons are polarized in our system, which is thus incontrovertible evidence for the intermediacy of a radical pair containing $\mathbf{D E S}^{\bullet+}$.

A more complete analysis can be given with Kaptein's rule [37] for the polarization phase $\Gamma_{i}$ of nucleus $i$ in a reaction product ( $\Gamma_{i}=+1$, absorption; $\Gamma_{i}=-1$, emission),

$$
\Gamma_{i}=\mu \times \varepsilon \times \operatorname{sign}(\Delta g) \times \operatorname{sign}\left(a_{i}\right)
$$

In Equation 3, the parameters $\mu$ and $\varepsilon$ characterize the reaction pathway ( $\mu$, precursor multiplicity leading to the radical pair: $\mu=+1$, triplet, $\mu=-1$, singlet; $\varepsilon$, radical-pair multiplicity from 
which the product is formed: $\varepsilon=+1$, singlet, $\varepsilon=-1$, triplet), and $\Delta g$ and $a_{i}$ are the pertinent magnetic properties of the radical pair $(\Delta g, g$-value difference of the two radicals, with the one containing nucleus $i$ taken first; $a_{i}$, hyperfine coupling constant of that nucleus in the radical). Together with the fact that the absolute CIDNP intensity of nucleus $i$ is approximately proportional to $a_{i}$ [7], this sign rule forms the basis for identifying a paramagnetic intermediate through the resulting polarization pattern.

The $g$ value of $\mathbf{D E S}^{\bullet+}$, regardless of whether it is present as a monomeric radical cation $(g=2.017$ [38]) or in its dimeric form $(g=2.011[25])$, is much larger than that of the pyranyl radical TPP $^{\bullet}(g=2.0031$ [39]), so $\Delta g$ must be positive for the sulfide protons; as stated above, the hyperfine coupling constant of $\mathbf{H}^{\alpha}$ is also positive. For the radical pair, reverse electron transfer is only possible in its singlet state $(\varepsilon=+1)$, because the triplet state of DES (281 kJ/mol [40]) lies even higher in energy than the initial excited singlet state of $\mathbf{T P P}^{+}(272 \mathrm{~kJ} / \mathrm{mol}$ [32]). The absorptive polarization for $\mathbf{H}^{\alpha}$ thus confirms the precursor multiplicity expected on the basis of the thermodynamic and photophysical data, i.e., triplet $(\mu=+1)$.

The absence of polarizations in the sensitizer is easily rationalized by the low hyperfine coupling constants in its radical TPP• (between 2.5 G and 0.4 G [39], as opposed to 18-20 G for $\mathbf{H}^{\alpha}$ in the monomeric [38] and $6.8 \mathrm{G}$ in the dimeric [25] radical cation of the sulfide) in conjunction with its complicated spectral habit, which causes the polarizations to be distributed among many resonances, with concomitant decrease in intensity of the individual peaks.

For tetrahydrothiophene THTP, the same polarization pattern (polarizations for $\mathbf{H}^{\alpha}$ only, i.e., CIDNP originating from radical pairs $\overline{\text { THTP }^{\bullet+} \text { TTP }^{\bullet}}$ ) and kinetic behavior of the polarizations was found, which is not surprising given the very similar thermodynamics (ionization potentials of DES and THTP, $8.42 \mathrm{eV}$ and $8.62 \mathrm{eV}$, respectively [41]) and magnetic parameters ${\text { (monomeric } \text { THTP }^{\bullet+}}^{\text {[38] }}, g=2.014, a_{\mathbf{H}} \alpha=20 \ldots 40 \mathrm{G}$; dimeric radical cation [25], $\left.g=2.010, a_{\mathbf{H}} \alpha=9.5 \mathrm{G}\right)$. The main difference between DES and THTP is a faster decay of the polarizations by the self-exchange in the case of the cyclic sulfide.

For the general reaction mechanism of electron-transfer-induced radical pair formation followed by exchange cancelation, the integrated rate law for the polarization $I_{\text {rel }}$ of the regenerated donor as a function of the time $t$ after the laser flash is biexponential $[17,19]$,
The fast component $k_{0}$ in Equation 4 is the decay rate of the excited state whereas the slow component $\kappa$,

$$
\kappa=k_{\mathrm{ex}} D_{0}+1 / T_{1}
$$

comprises the effects of self-exchange (rate constant, $k_{\text {ex }}$; donor concentration, $D_{0}$ ) and nuclear spin relaxation in the free radicals (relaxation time, $T_{1}$ ). The latter spoils a perfect cancelation of geminate and escape polarizations, so is responsible for the residual magnetization at long times after the flash. Bimolecular recombination of the free radicals, which would lead to an additional term in Equation 5 [17], usually is of marginal importance only [19], and was neglected in our analysis because no dependence of $\kappa$ on the laser intensity, and hence on the radical concentration, was observed.

Owing to technical reasons, NMR pulses often cannot be made infinitely short on the timescale of the polarization changes, and sampling time-dependent CIDNP with such "long" pulses (duration, $\tau$ ) then results in a convolution of pulse action and kinetics [42]. Under our experimental conditions, this convolution is tantamount to simply multiplying each exponential term $\exp (-k t)$ in Equation 4, where $k$ is $k_{0}$ or $\kappa$, with a constant factor,

$$
\exp (-k t) \rightarrow \frac{1-\exp (-k \tau)}{k \tau} \exp (-k t)
$$

The changing of the weights of the two exponentials in Equation 4 by Equation 6 is the reason why the polarizations seen in Figure 1 do not start from zero at time zero after the laser flash.

Eyring plots of the exchange rate constants so obtained for DES and THTP can be found in Figure 2, and Table 1 lists the activation parameters resulting from these plots. With DES, experiments at different concentrations gave slight systematic displacements of the regression lines, corresponding to about $20 \%$ lower apparent values of $k_{\mathrm{ex}}$ when the amount of sulfide was halved. We tentatively ascribe this to the dimerization of the radical cations discussed below; because of our high radical concentrations, the apparent depletion of DES by this complexation is higher for smaller substrate concentrations. On these grounds, we regard the intercept in Figure 2, and thus the activation entropy in Table 1, as a lower limit in the case of DES.

The activation enthalpies are unusually small, but much more striking are the strongly negative activation entropies because it

$$
I_{\text {rel }}=A\left[\left(1-1 /\left(\kappa T_{1}\right)\right) \exp (-\kappa t)-\left(1-1 /\left(k_{0} T_{1}\right)\right) \exp \left(-k_{0} t\right)+\left(k_{0}-\kappa\right) /\left(k_{0} \kappa T_{1}\right)\right]
$$




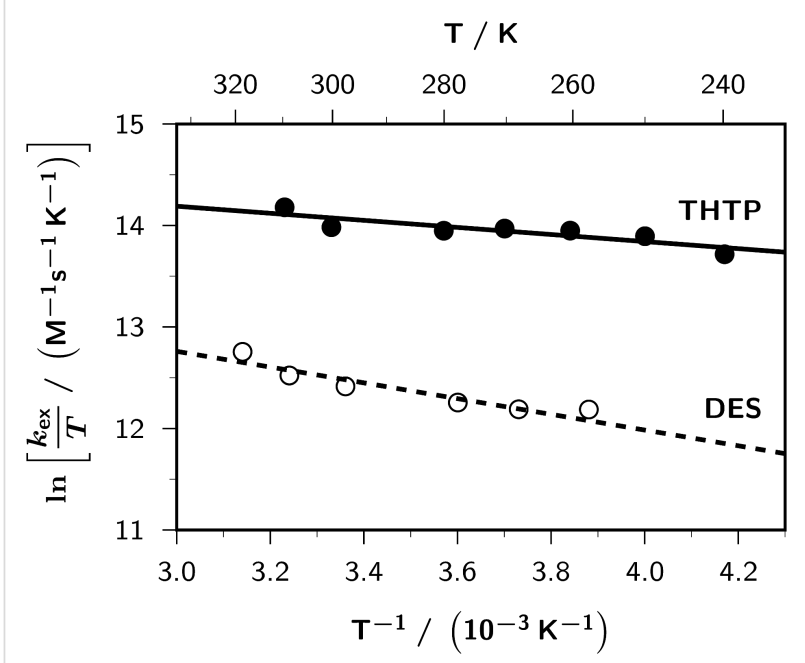

Figure 2: Eyring plots of the self-exchange rate constants $k_{\text {ex }}$ for DES (open circles and broken line; linear regression, $15.08-775 / T$, $r^{2}=0.94$ ) and THTP (filled circles and solid line; linear regression, $15.24-349 / T, r^{2}=0.88$ ) in acetonitrile- $d_{3}$; sensitizer TTP ${ }^{+}$. For all other experimental parameters and further explanation, see Figure 1 and text.

Table 1: Activation parameters (enthalpy $\Delta H_{0}^{\neq}$, entropy $\Delta S_{0}^{\neq}$, and free energy at room temperature $\Delta G_{0}^{\neq}(298 \mathrm{~K})$ ) for the self-exchange reactions of diethyl sulfide DES and tetrahydrothiophene THTP with their respective radical cations in acetonitrile.

\begin{tabular}{lccc} 
Sulfide & $\begin{array}{c}\Delta H_{0}^{\neq} \\
(\mathrm{kJ} / \mathrm{mol})\end{array}$ & $\begin{array}{c}\Delta S_{0}^{\neq} \\
\left(\mathrm{J} \mathrm{K}^{-1} \mathrm{~mol}^{-1}\right)\end{array}$ & $\begin{array}{c}\Delta G_{0}^{\neq}(298 \mathrm{~K}) \\
(\mathrm{kJ} / \mathrm{mol})\end{array}$ \\
\hline DES & 6.4 & -72.1 & 27.9 \\
THTP & 2.9 & -70.9 & 24.0
\end{tabular}

is well known that outer-sphere electron transfer of organic substrates in polar solvents is usually accompanied by small positive activation entropies [43] (e.g., our recent study on triisopropylamine [20] gave an activation entropy of $+7.6 \mathrm{JK}^{-1} \mathrm{~mol}^{-1}$ ). Our experimental values indicate transitions states that are much more ordered than usual, which is strong evidence that in these systems the self-exchange does not involve the monomeric radical cations $\mathbf{D}^{\bullet+}$ but the dimeric ones (D $\therefore$ D $)^{+}$, for which the formation of the new two-center three-electron bond greatly restricts the number of possible orientations of the reacting molecules.

Because of the considerable thermodynamic driving force [24], the initial formation of the dimeric cations must be much faster than the self-exchange, so cannot be captured by our timeresolved CIDNP experiments. It might even occur during the spin-correlated life of the radical pairs without being detectable by the polarization pattern because neither the $g$-value difference nor the distribution of unpaired spin density between the $\alpha$ and $\beta$ protons changes in it, as discussed above.

Despite the obvious deviation of such an associative-dissociative process from a simple outer-sphere electron transfer, its experimental free energy of activation $\Delta G_{0}^{\neq}$seems to be well reproduced by the Marcus theory in our case. That theory [1,2] predicts $\Delta G_{0}^{\neq}$to be one quarter of the reorganization energy $\lambda$, which is the hypothetical energy needed for all the geometrical changes occurring in the reaction, but without actually transferring the electron. This includes converting the geometry of the nonradical species into that of the radical species, and vice versa (inner reorganization energy $\lambda_{\mathrm{i}}$ ), as well as rearranging the solvent shell to accommodate the charge distribution of the respective other side of the reaction equation (outer reorganization energy $\lambda_{0}$ ).

In the majority of cases, $\lambda_{\mathrm{i}}$, which is usually calculated from the force constants and geometry differences of the reactants $[1,2]$, constitutes but a small correction to the total reorganization energy, on the order of $10 \%$ [44]. At first glance, one would expect the situation to differ for our associative-dissociative electron transfers. For DES, however, all bond lengths and angles in the radical cation, regardless of monomeric or dimeric (except, of course, for the bond between the two sulfur atoms, which is only present in the dimer), and in the parent compound are extremely similar, with the maximum changes during the reaction lying well below $0.01 \AA$ [45]; using the known valence force field for DES [46], one thus arrives at utterly negligible effects on $\lambda_{\mathrm{i}}$ from all these bonds. As regards the sulfur-sulfur bond, from the shape of its associated optical absorption band its potential energy curve was concluded [24] to be quite broad and shallow, so it seems reasonable to identify its contribution to $\lambda_{i}$ with the free energy of the monomer-dimer equilibrium, which can in turn be estimated from the reported equilibrium constants [24] to lie around $20 \mathrm{~kJ} / \mathrm{mol}$. Because that is only about a fifth of the experimental total reorganization energy, the uncertainties of the approximation cannot play an important role; on the other hand, $\lambda_{0}$ obviously also remains the dominant term by far, also for this reaction type.

The solvent dependence of $\Delta G_{0}^{\neq}$would allow a separation of $\lambda_{\mathrm{i}}$ and $\lambda_{0}$, as only the latter is polarity dependent. Unfortunately, however, a variation of the solvent proved infeasible. In very nonpolar media, $\mathbf{T} \mathbf{P} \mathbf{P}^{+}$is not adequately soluble; in protic or nucleophilic solvents, the samples developed a brownish color after only a few laser shots and the CIDNP intensity decreased drastically, so time-resolved measurements could not be performed; in yet further solvents, such as DMSO, the 
residual signal of the solvent interfered with an exact determination of the polarization intensities, which are rather small in these systems owing to the large $g$-value differences.

Modeling the reacting molecules as spheres with diameters $a_{1}$ and $a_{2}$ and a distance $d$ between them, Marcus [1,2] has derived an expression describing $\lambda_{0}$ for the self-exchange between a neutral molecule and an ion of charge $z$,

$$
\lambda_{\mathrm{o}}=\frac{N_{\mathrm{A}}(z e)^{2}}{4 \pi \varepsilon_{0}} \times\left(\frac{1}{n^{2}}-\frac{1}{\varepsilon}\right) \times\left(\frac{1}{a_{1}}+\frac{1}{a_{2}}-\frac{1}{d}\right)
$$

with $N_{A}, e$, and $\varepsilon_{0}$ being Avogadro's number, the electron charge, and the vacuum permittivity. The second term of the product in Equation 7, the so-called polarity factor, characterizes the ability of the solvent to follow the charge redistribution during the exchange ( $n$, refractive index; $\varepsilon$, relative permittivity); for acetonitrile, its value is 0.527 at room temperature. Several groups $[47,48]$ including ours [49] have extended Equation 7 to multisphere models to take into account the charge distribution in the molecules.

In the associative-dissociative self-exchange between $\mathbf{D}$ and (D $\therefore$ D $)^{+}$, the charge distribution on one half of the dimer remains unchanged, so effectively $z$ amounts to $1 / 2$ only. Furthermore, there is no delocalization in our aliphatic substrates; hence, it seems natural to assume the exchanged charge to be basically localized on two of the three sulfur atoms. In this limit, the multisphere model again turns into Equation 7, with $a_{1}$ and $a_{2}$ being identical and representing the diameter of the charge cloud around each sulfur. There exists no clear criterion for deciding the value of $a_{1,2}$, but choosing the length of the sulfur-sulfur bond in the dimer (2.9 $\AA$ [45]) as a reasonable approximation of this quantity and using the data of Table 1 as well as $\lambda_{\mathrm{i}}$ estimated above, one arrives at physically very reasonable distances $d$ of $5.3 \AA$ and $3.7 \AA$ for DES and THTP. In the latter case, a closer approach is in line with expectation because the restricted geometric freedom and more compact configuration of the aliphatic substituents leave the sulfur atom more accessible; furthermore, the obtained reaction distance is practically equal to twice the van-der-Waals radius of sulfur [50], so is also very plausible.

\section{Conclusion}

The present study has shed light on an unusual variant of electron self-exchange, namely, an associative-dissociative mechanism involving dimeric radical cations and monomeric parent compounds. It serves to illustrate the power of CIDNP to isolate certain steps within a complex reaction scheme and investigate their kinetics. Not only does it permit the identification of early, short-lived paramagnetic intermediates by freezing their spindensity distribution as a pattern of nuclear spin polarizations, but those polarizations then provide convenient labels that establish a connection with subsequent species on the reaction coordinate and, specific to this work, make the two sides of a self-exchange reaction distinguishable, as to allow one to measure its rate.

\section{Experimental}

All chemicals were commercially available. DES was purified by distillation, $\mathbf{T P P}^{+}$and THTP were used as received, the solvent acetonitrile- $d_{3}$ was dried to a water content below $5 \times 10^{-4} \mathrm{M}$ in a dedicated apparatus [51]. Oxygen was removed by bubbling nitrogen through the ice-cooled (to reduce losses of sulfide and solvent) solutions; the samples were then sealed. The final concentration of the sulfide in the samples was determined by NMR spectroscopy. Time-resolved ${ }^{1} \mathrm{H}(250 \mathrm{MHz})$ CIDNP measurements were performed on a Bruker WM 250 NMR spectrometer with a special probe [17] that provided illumination of the samples from the side. All unchanging background magnetization was removed by presaturation pulse sequences [52]. Excitation was done with a Lambda Physik EMG 101 excimer laser (medium, $\mathrm{XeCl}$; wavelength, $308 \mathrm{~nm}$; pulse width, $15 \mathrm{~ns}$ ) triggered by the computer of the spectrometer. The probe was thermostated to $\pm 0.3 \mathrm{~K}$. Quantum mechanical calculations were carried out with the Gaussian 09 package [53] using the AM1 Hamiltonian.

\section{References}

1. Marcus, R. A. Annu. Rev. Phys. Chem. 1964, 15, 155-196. doi:10.1146/annurev.pc.15.100164.001103

2. Marcus, R. A.; Sutin, N. Biochim. Biophys. Acta, Rev. Bioenerg. 1985, 811, 265-322. doi:10.1016/0304-4173(85)90014-X

3. Burri, J.; Fischer, H. Chem. Phys. 1992, 161, 429-435. doi:10.1016/0301-0104(92)80158-R

4. Grampp, G. Spectrochim. Acta, Part A 1998, 54, 2349-2358. doi:10.1016/S1386-1425(98)00215-7

5. Steiner, U. E.; Ulrich, T. Chem. Rev. 1989, 89, 51-147. doi:10.1021/cr00091a003

6. Goez, M. Adv. Photochem. 1997, 23, 63-163. doi:10.1002/9780470133545.ch2

7. Goez, M. Annu. Rep. NMR Spectrosc. 2009, 66, 77-147. doi:10.1016/S0066-4103(08)00403-1

8. Goez, M. In Carbon-Centered Free Radicals and Radical Cations: Structure, Reactivity, and Dynamics; Forbes, M. D., Ed.; Wiley Series on Reactive Intermediates in Chemistry and Biology, Vol. 3; 2010; pp 185-204.

9. Berliner, L. J.; Bagryanskaya, E. In Multifrequency Electron Paramagnetic Resonance; Misra, S. K., Ed.; Wiley-VCH: Weinheim, 2011; pp 947-992.

10. Goez, M. Top. Curr. Chem. 2012. doi:10.1007/128_2012_348.

11. Kavarnos, G. J.; Turro, N. J. Chem. Rev. 1986, 86, 401-449. doi:10.1021/cr00072a005 
12. Closs, G. L.; Sitzmann, E. V. J. Am. Chem. Soc. 1981, 103, 3217-3219. doi:10.1021/ja00401a052

13. Schäublin, S.; Wokaun, A.; Ernst, R. R. J. Magn. Reson. 1977, 27, 273-302. doi:10.1016/0022-2364(77)90077-4

14. Closs, G. L.; Miller, R. J. J. Am. Chem. Soc. 1979, 101, 1639-1641. doi:10.1021/ja00500a068

15. Goez, M.; Kuprov, I.; Hore, P. J. J. Magn. Reson. 2005, 177, 139-145. doi:10.1016/j.jmr.2005.06.017

16. Goez, M.; Kuprov, I.; Mok, K. H.; Hore, P. J. Mol. Phys. 2006, 104, 1675-1686. doi:10.1080/00268970600634431

17. Goez, M. Chem. Phys. 1990, 147, 143-154 doi:10.1016/0301-0104(90)85030-Z

18. Goez, M. Z. Phys. Chem. 1990, 169, 123-132. doi:10.1524/zpch.1990.169.Part_2.123

19. Goez, M.; Eckert, G. Ber. Bunsen-Ges. Phys. Chem. 1991, 95 , 1179-1186. doi:10.1002/bbpc.19910951004

20. Goez, M.; Frisch, I.; Sartorius, I. Beilstein J. Org. Chem. 2013, 9 , 437-446. doi:10.3762/bjoc.9.46

21. Goez, M.; Sartorius, I. J. Am. Chem. Soc. 1993, 115, 11123-11133. doi:10.1021/ja00077a009

22. Goez, M.; Sartorius, I. Chem. Ber. 1994, 127, 2273-2276. doi:10.1002/cber.1491271128

23. Goez, M.; Sartorius, I. J. Phys. Chem. A 2003, 107, 8539-8546. doi:10.1021/jp030244g

24. Asmus, K. D. Acc. Chem. Res. 1979, 12, 436-442. doi:10.1021/ar50144a003

25. Werst, D. W. J. Phys. Chem. 1992, 96, 3640-3646. doi:10.1021/j100188a017

26. Vogtherr, M.; Goez, M. J. Inf. Rec. 1998, 24, 23-27.

27. Ando, W. Sulfur Rep. 1981, 1, 147-207. doi:10.1080/17415998109408001

28. Mönig, J.; Göbl, M.; Asmus, K.-D. J. Chem. Soc., Perkin Trans. 2 1985, 647-651. doi:10.1039/p29850000647

29. Goez, M.; Rozwadowski, J.; Marciniak, B. J. Am. Chem. Soc. 1996, 118, 2882-2891. doi:10.1021/ja9536678

30. Aizawa, T.; Sakata, T.; Itoh, S.; Maeda, K.; Azumi, T. Chem. Phys. Lett. 1992, 195, 16-20. doi:10.1016/0009-2614(92)85903-N

31. Saeva, F. D.; Olin, G. R. J. Am. Chem. Soc. 1980, 102, 299-303. doi:10.1021/ja00521a047

32. Miranda, M. A.; Garcia, H. Chem. Rev. 1994, 94, 1063-1089. doi:10.1021/cr00028a009

33. Bonesi, S. M.; Manet, I.; Freccero, M.; Fagnoni, M.; Albini, A. Chem.-Eur. J. 2006, 12, 4844-4857. doi:10.1002/chem.200501144

34. Bonesi, S. M.; Carbonell, E.; Garcia, H.; Fagnoni, M.; Albini, A. Appl. Catal., B 2008, 79, 368-375. doi:10.1016/j.apcatb.2007.10.037

35. Banu, I. S.; Ramamurthy, P. J. Photochem. Photobiol., A 2009, 201, 175-182. doi:10.1016/j.jphotochem.2008.10.019

36. Roth, H. D.; Manion, M. L. J. Am. Chem. Soc. 1975, 97, 6886-6888. doi:10.1021/ja00856a060

37. Kaptein, R. J. Chem. Soc. D 1971, 732-733. doi:10.1039/C29710000732

38. Rao, D. N. R.; Symons, M. C. R.; Wren, B. W. J. Chem. Soc., Perkin Trans. 2 1984, 1681-1687. doi:10.1039/p29840001681

39. Degani, I.; Lunazzi, L.; Pedulli, G. F. Mol. Phys. 1968, 14, 217-222. doi:10.1080/00268976800100241

40. Shiraishi, Y.; Koizumi, H.; Hirai, T. J. Phys. Chem. B 2005, 109 , 8580-8586. doi:10.1021/jp045639u
41. NIST Chemistry WebBook; NIST Standard Reference Database Number 69; Linstrom, P., Mallard, W., Eds.; National Institute of Standards and Technology, Gaithersburg MD, 20899. http://webbook.nist.gov (accessed April 22, 2013).

42. Goez, M. Chem. Phys. Lett. 1990, 165, 11-14. doi:10.1016/0009-2614(90)87003-A

43. Ghorai, P. K.; Matyushov, D. V. J. Phys. Chem. A 2006, 110, 8857-8863. doi:10.1021/jp056261i

44. Jensen, B. S.; Ronlán, A.; Parker, V. D. Acta Chem. Scand., Ser. B 1975, 29, 394-397. doi:10.3891/acta.chem.scand.29b-0394

45. Deng, Y.; Illies, A. J.; James, M. A.; McKee, M. L.; Peschke, M. J. Am. Chem. Soc. 1995, 117, 420-428. doi:10.1021/ja00106a048

46. Scott, D. W.; El-Sabban, M. Z. J. Mol. Spectrosc. 1969, 30, 317-337. doi:10.1016/0022-2852(69)90267-7

47. Peover, M. E.; Powell, J. S. J. Electroanal. Chem. Interfacial Electrochem. 1969, 20, 427-433. doi:10.1016/S0022-0728(69)80172-5

48. Grampp, G.; Jaenicke, W. Ber. Bunsen-Ges. Phys. Chem. 1984, 88, 325-334. doi:10.1002/bbpc.19840880403

49. Goez, M. Z. Phys. Chem. 1990, 169, 133-145. doi:10.1524/zpch.1990.169.Part_2.133.

50. Bondi, A. J. Phys. Chem. 1964, 68, 441-451. doi:10.1021/j100785a001

51. Goez, M. J. Magn. Reson. 1998, 135, 14-16. doi:10.1006/jmre.1998.1504

52. Goez, M.; Mok, K. H.; Hore, P. J. J. Magn. Reson. 2005, 177, 236-246. doi:10.1016/j.jmr.2005.06.015

53. Gaussian 09, Revision A.1; Gaussian, Inc.: Wallingford, CT, 2009.

\section{License and Terms}

This is an Open Access article under the terms of the Creative Commons Attribution License (http://creativecommons.org/licenses/by/2.0), which permits unrestricted use, distribution, and reproduction in any medium, provided the original work is properly cited.

The license is subject to the Beilstein Journal of Organic Chemistry terms and conditions:

(http://www.beilstein-journals.org/bjoc)

The definitive version of this article is the electronic one which can be found at: doi:10.3762/bjoc. 9.164 